\title{
ESTUDO DO EMPREGO DE MATERIAIS SINTERIZADOS SUBMETIDOS A EXTRUSÃO A MORNO*
}

\author{
Matheus Fortes Stiborski ${ }^{1}$ \\ Alana Meregalli ${ }^{2}$ \\ André Carvalho Tavares ${ }^{3}$ \\ Diego Pacheco Wermuth ${ }^{4}$ \\ Silmar do Nascimento Castro ${ }^{5}$ \\ Lirio Schaeffer ${ }^{6}$
}

\section{Resumo}

O emprego de materiais sinterizados para conformação massiva permite a utilização de forças menores permitindo que resultados similares sejam alcançados com maquinas de potência menor, outra vantagem é que o blank utilizado para esse processo não contém tensões residuais advindas do processo anterior. Para o processo foi necessário o levantamento das curvas de compressibilidade do material a ser estudado (pó de ferro puro), análise das propriedades do material sinterizado e posteriormente extrusão do material e caracterização do mesmo..

Palavras-chave: Metalurgia do pó; Extrusão; Ferro puro; Propriedades mecânicas.

\section{EMPLOYMENT MATERIALS SINTERING STUDY APPLIED IN WARM EXTRUSION}

\section{Abstract}

The use of sintered materials for massive conformation allows the use of smaller forces allowing similar results are achieved with lower power machines, another advantage is that the blank used for this process contains no residual stresses resulting from the previous process. For the process it was necessary to lift curves of the compressibility of the material to be studied ( pure iron powder), analysis of the properties of the sintered material and then extruding the material and characterization of the same.

Keywords: Powder metallurgy; Extrusion; Pure Iron; Mechanical Properties.

1 Graduando em Engenharia Metalúrgica na Universidade Federal do Rio Grande do Sul, Bolsista de Iniciação Tecnológica do Laboratório de Transformação Mecânica (LdTM), Porto Alegre, RS, Brasil.

2 Graduanda em Engenharia Metalúrgica na Universidade Federal do Rio Grande do Sul, Porto Alegre, RS, Brasil.

3 Doutorando do Programa de Pós-Graduação em Engenharia de Minas, Metalúrgica e Materiais (PPGE3M). Laboratório de Transformação Mecânica. (LdTM), Depto. de Metalurgia, UFRGS, Porto Alegre, RS, Brasil.

4 Doutorando do Programa de Pós-Graduação em Engenharia de Minas, Metalúrgica e Materiais (PPGE3M). Laboratório de Transformação Mecânica. (LdTM), Depto. de Metalurgia, UFRGS, Porto Alegre, RS, Brasil.

5 Graduando em Engenharia Metalúrgica na Universidade Federal do Rio Grande do Sul, Bolsista de Iniciação Tecnológica do Laboratório de Transformação Mecânica (LdTM), Porto Alegre, RS, Brasil.

6 Engenheiro Mecânico, Doutor em Engenharia, Professor na Universidade Federal do Rio Grande do Sul, Diretor do Laboratório de Transformação Mecânica (LdTM), Porto Alegre, RS, Brasil. 


\section{INTRODUÇÃO}

Neste artigo serão confeccionadas peças de ferro puro através do processo de Metalurgia do Pó convencional. Este processo metalúrgico consiste em conformar os pós constituintes da liga em peças resistentes, comprimindo estes pós no interior de uma ferramenta com formato desejado obtendo um "compactado à verde" por pressão mecânica que, posteriormente, será sinterizado. Peças fabricadas por metalurgia do pó possuem certa porosidade, que pode ser controlada através de fatores como pressão de compactação, temperatura e tempo de sinterização, tamanho e forma das partículas do pó, etc. A peça pronta apresenta bom acabamento superficial e adequada tolerâncias dimensionais (1). A metalurgia do pó tem a finalidade de transformar sem fusão efetiva, pós metálicos ou não metálicos usando pressão para compactação e calor, através de um tratamento térmico de sinterização, que se realiza a temperatura inferior ao ponto de fusão do material base do componente ou peça, permitindo obtê-los na sua forma final ou próximo dela ('near-net or net-shape') (2).

Este corpo obtido ao término do processo de metalurgia do pó será utilizado como pré-forma para formar peças forjadas com dimensionamento final superiores as obtidas a partir de pré-formas oriundas de outros processos de fabricação, além, de melhores propriedades mecânicas e metalúrgicas devido a homogeneidade da distribuição dos grãos. O padrão de deformação durante a extrusão de corpos sinterizados é diferente do convencional, pois, as características do material poroso passando por deformação são de fundamental importância, pois, a eliminação dos poros, iniciada no inicio da deformação plástica, ocasiona uma mudança de densidade

\section{MATERIAIS E MÉTODOS}

Inicialmente foram compactadas amostras em diferentes pressões com o intuito de realizar uma curva de compressão do pó metálico (Ferro Puro ASC 100,29). Foram traçadas com pressões variando de 0 a $1000 \mathrm{MPa}$, como pode ser visto na Figura 1.

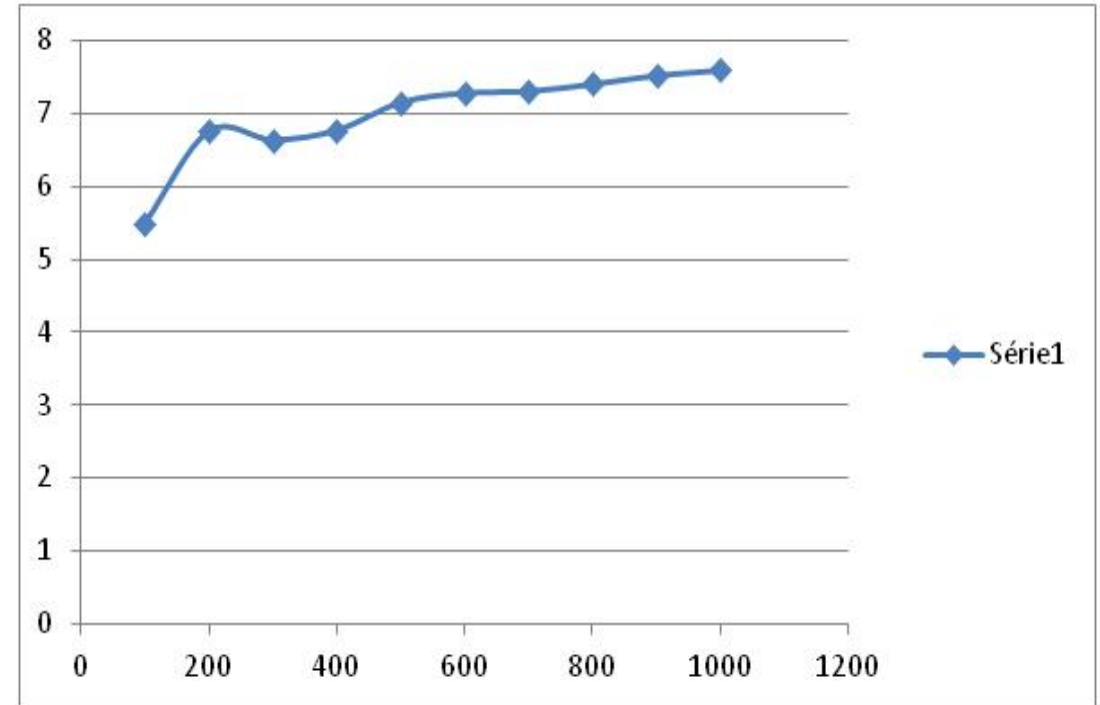

Figura 1. Curva de Compressão. 
As amostras confeccionadas foram sinterizadas em um forno tubular a uma temperatura de $1150^{\circ} \mathrm{C}$ com uma taxa de aquecimento de $10^{\circ} \mathrm{C}$. As amostras sinterizadas estão exibidas na figura 2 .

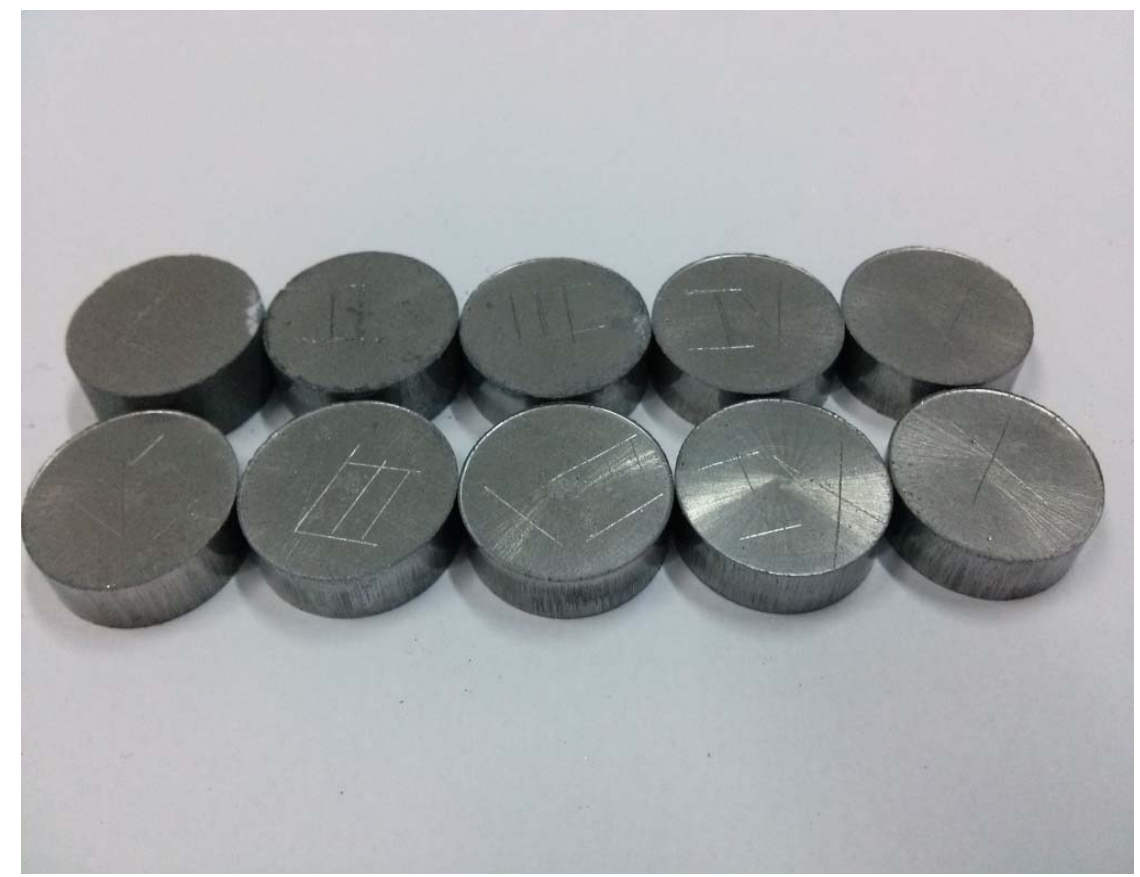

Figura 2 - Peças sinterizadas de Fe Puro

Após a sinterização as amostras foram encaminhadas para analises de dureza, microdureza, metalografia e medições de densidade das amostras.

\section{RESULTADOS E DISCUSSÃO}

A peça confeccionada com a pressão de $1000 \mathrm{MPa}$, que mostrou a estabilização da curva e das propriedades, foi levada para o processo de conformação por extrusão. A matriz de extrusão foi colocada em um forno por 120 min à temperatura de $600^{\circ} \mathrm{C}$, as peças foram mergulhadas em lubrificante a base de grafite, deixando por $30 \mathrm{~min}$ para reestabilização térmica, sendo encaminhado ao término deste período a uma prensa de 40 ton para execução do processo de extrusão.

A partir do processo de extrusão foi provocada uma deformação plástica do material ocasionando o encruamento das amostras, ocorrendo o fechamento dos poros e aumento das propriedades mecânicas.

As amostras compactadas e sinterizadas foram submetidas a medição de microdureza. Os valores obtidos estão descritos na tabela 1. 
Tabela 1. Microdurezas antes da extrusão

\section{MICRODUREZA VICKERS}

\begin{tabular}{|c|c|c|c|c|c|c|c|c|c|}
\hline $\begin{array}{c}\text { Amostra } \\
\text { I }\end{array}$ & $\begin{array}{c}\text { Amostra } \\
\text { II }\end{array}$ & $\begin{array}{c}\text { Amostra } \\
\text { III }\end{array}$ & $\begin{array}{c}\text { Amostra } \\
\text { IV }\end{array}$ & $\begin{array}{c}\text { Amostra } \\
\text { V }\end{array}$ & $\begin{array}{c}\text { Amostra } \\
\text { VI }\end{array}$ & $\begin{array}{c}\text { Amostra } \\
\text { VII }\end{array}$ & $\begin{array}{c}\text { Amostra } \\
\text { VIII }\end{array}$ & $\begin{array}{c}\text { Amostra } \\
\text { IX }\end{array}$ & $\begin{array}{c}\text { Amostra } \\
\mathbf{X}\end{array}$ \\
\hline 159,24 & 85,99 & 82,54 & 58,53 & 83,86 & 75,53 & 103,88 & 105,81 & 118,78 & 107,72 \\
\hline 136,82 & 103,69 & 82,36 & 76,26 & 86,74 & 85,35 & 122,07 & 96,33 & 124,86 & 119,21 \\
\hline 204,49 & 82,58 & 83,46 & 58,24 & 86,37 & 85,86 & 115,96 & 101,74 & 110,06 & 112,45 \\
\hline 187,17 & 81,16 & 83,15 & 72,71 & 78,15 & 83,33 & 116,48 & 99,59 & 119,43 & 118,89 \\
\hline \multicolumn{10}{|c|}{ MÉDIAS } \\
\hline 171,93 & 88,36 & 82,88 & 66,44 & 83,78 & 82,52 & 114,60 & 100,87 & 118,28 & 114,57 \\
\hline
\end{tabular}

Ao analisarmos as microdurezas, observa-se que a amostra IX compactada a 900 $\mathrm{MPa}$ apresentou a maior dureza, seguida da amostra VII e X.

Os corpos de prova sinterizados para extrusão, exibidos na figura 3, foram confeccionados com $600 \mathrm{MPa}$ devido as limitações da prensa utilizada e as dimensões da matriz de extrusão disponível.

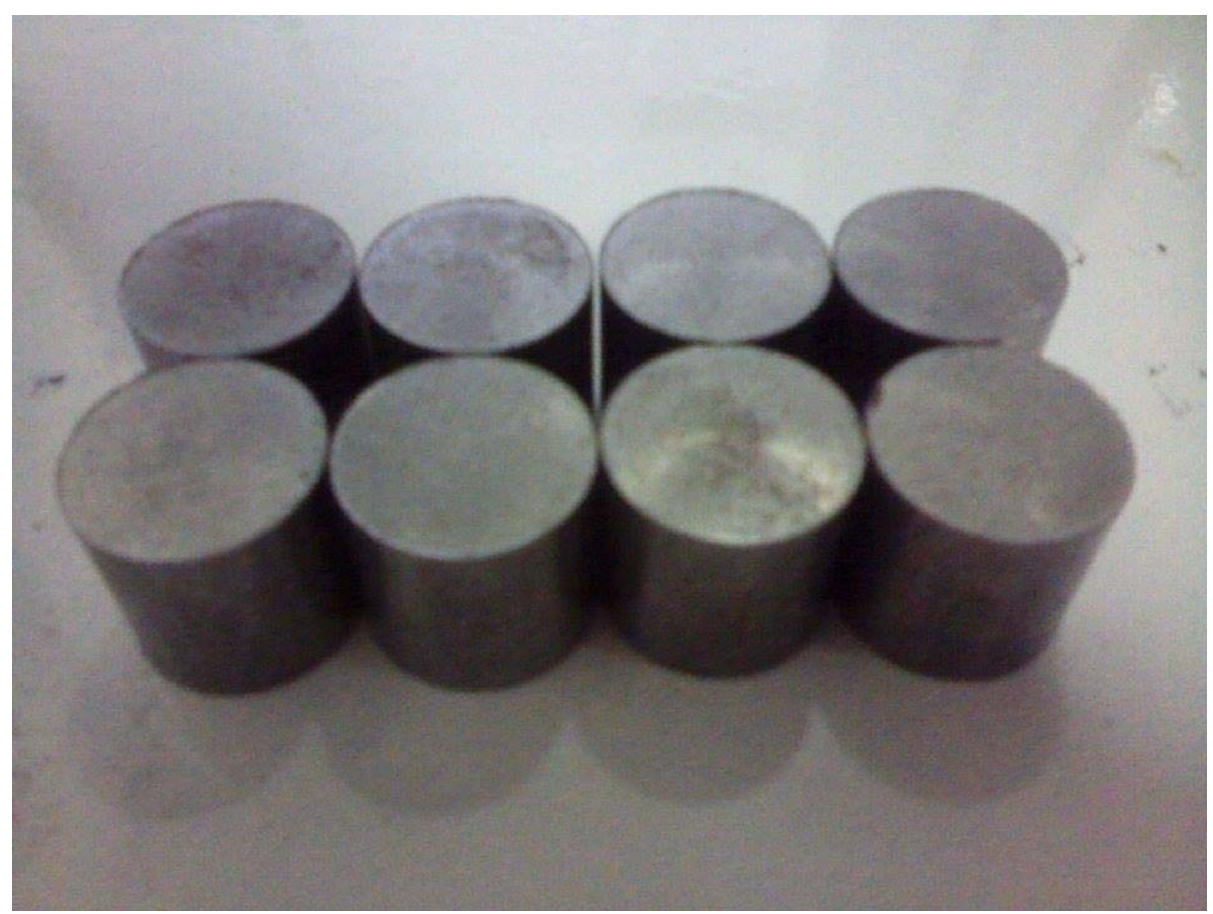

Figura 3 - Peças sinterizadas para extrusão

As peças extrudadas, exibidas na figura 4 , foram submetidas a analise metalografica e medições de dureza. 


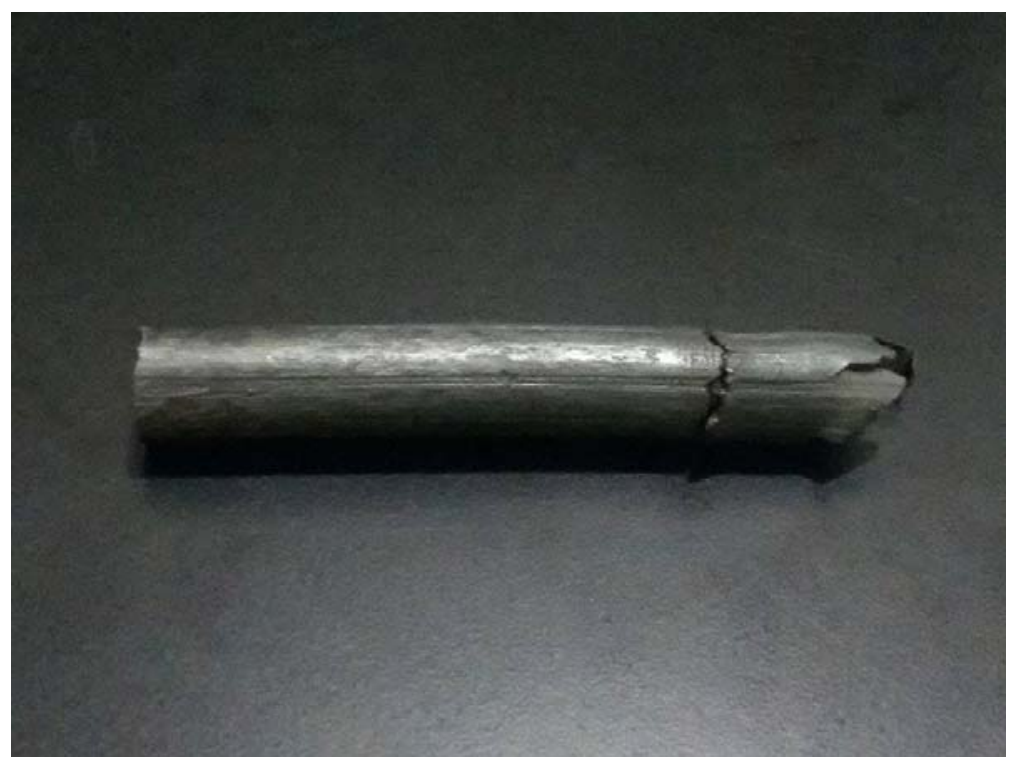

Figura 4 - Peças sinterizadas para extrusão

Após a extrusão foi analisada a dureza das peças, sendo estas apresentadas pela tabela 2.

Tabela 2. Microdurezas após a extrusão

\begin{tabular}{|c|c|}
\hline \multicolumn{2}{|c|}{ MICRODUREZA VICKERS } \\
\hline Corte Amostra Extrudada & Corte Transversal Amostra Extrudada \\
\hline 147,32 & 154,93 \\
\hline 140,1 & 146,57 \\
\hline 141,75 & 143,43 \\
\hline 142,44 & 155,1 \\
\hline 140,65 & 149,84 \\
\hline 141,18 & 150,45 \\
\hline \multicolumn{2}{|c|}{ MÉDIAS } \\
\hline 142,24 & 150,0533333 \\
\hline \multicolumn{2}{|c|}{ Extremidades } \\
\hline 152,28 & 156,37 \\
\hline 155,25 & 160,29 \\
\hline 153,83 & 157,33 \\
\hline 161,46 & 160,46 \\
\hline 151,97 & 151,67 \\
\hline 172,43 & 157,5 \\
\hline 164,36 & 160,79 \\
\hline 171,34 & 153,83 \\
\hline 152,89 & 173,76 \\
\hline 156,84 & 153,67 \\
\hline 156,05 & 159,8 \\
\hline 150,58 & 157,66 \\
\hline \multicolumn{2}{|c|}{ MÉDIAS } \\
\hline 158,2733333 & 158,5941667 \\
\hline
\end{tabular}


Foi analisado a metalografia das peças extrudadas, onde notou-se mudança também no tamanho de grão do material conforme pode ser visto na Figura 5.

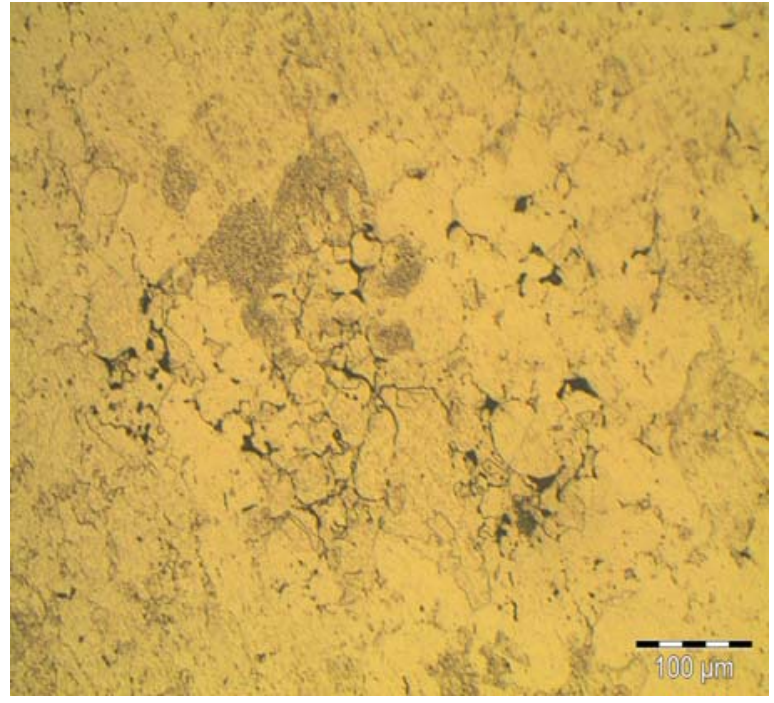

(a)

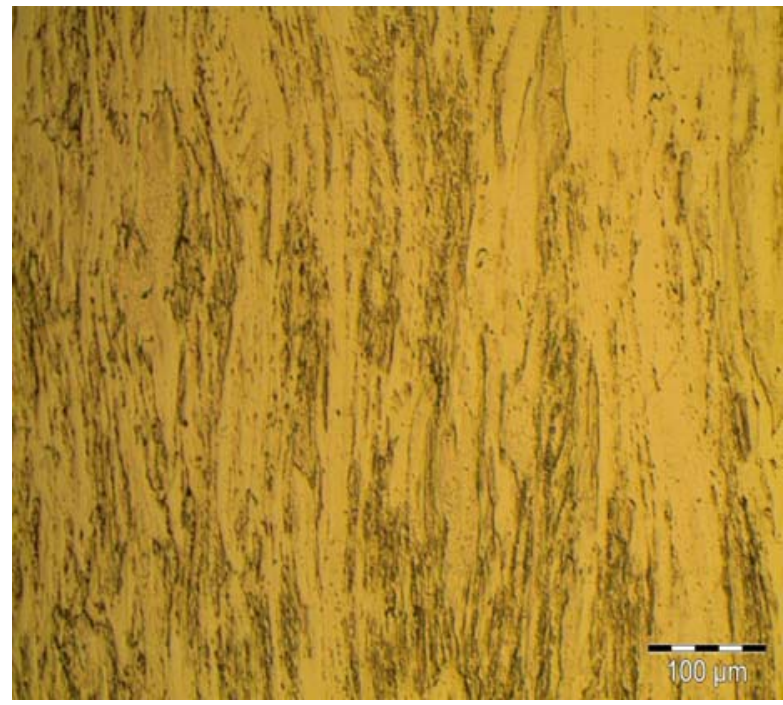

(b)

Figura 5 - Comparação do grão: antes(a) e depois (b) do processo de extrusão

\section{CONCLUSÃO}

O processo de extrusão é viável após a sinterização das peças devido ao possível aumento das propriedades mecânicas e físicas através do encruamento do material, conforme pode ser analisado nas medidas de microdureza vickers. Através da analise metalográfica foi possível verificar diminuição no tamanho de grão, bem como alinhamento no sentido de orientação cristalográfica. A utilização de peças obtidas por metalurgia do pó com posterior conformação possibilita uma diminuição da energia demandada para obter o processo comparado a amostras laminadas fundidas ou extrudadas.

\section{Agradecimentos}

À Universidade Federal do Rio Grande do Sul, principalmente ao Laboratório de Transformação Mecânica (LdTM) pela disponibilização dos recursos materiais e tecnológicos utilizados na elaboração deste trabalho. Aos órgãos CAPES e CNPq pelo apoio a projetos e investimento na área da pesquisa.

\section{REFERÊNCIAS}

1 DIETER, G. E. Mechanical Metallurgy. Vol. 3, ed. McGraw- Hill, London, United Kingdom, 1988

2 DONNELL, G. O. LOONEY, L. Production of aluminium matrix composite components using conventional PM technology. Materials Science and Engineering: A, vol. 303, p. 292-301, 2001. 